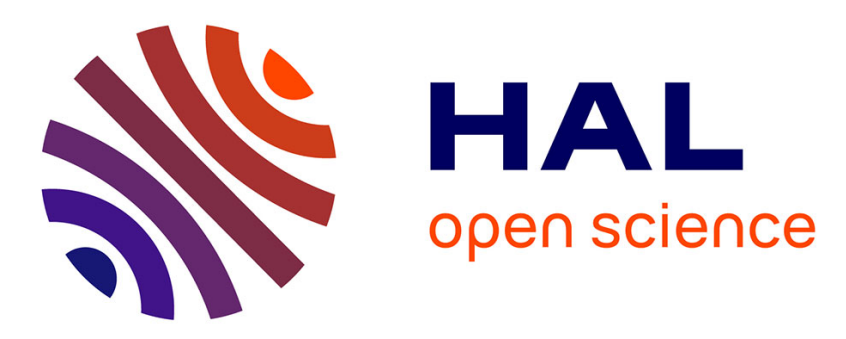

\title{
Modeling of electrochemically generated bubbly flow under buoyancy-driven and forced convection
}

\author{
Jonathan Schillings, Olivier Léon Doche, Jonathan Deseure
}

\section{To cite this version:}

Jonathan Schillings, Olivier Léon Doche, Jonathan Deseure. Modeling of electrochemically generated bubbly flow under buoyancy-driven and forced convection. International Journal of Heat and Mass Transfer, 2015, 85, pp.292-299. 10.1016/j.ijheatmasstransfer.2015.01.121 . hal-02545876

\section{HAL Id: hal-02545876 https://hal.science/hal-02545876}

Submitted on 28 Apr 2020

HAL is a multi-disciplinary open access archive for the deposit and dissemination of scientific research documents, whether they are published or not. The documents may come from teaching and research institutions in France or abroad, or from public or private research centers.
L'archive ouverte pluridisciplinaire HAL, est destinée au dépôt et à la diffusion de documents scientifiques de niveau recherche, publiés ou non, émanant des établissements d'enseignement et de recherche français ou étrangers, des laboratoires publics ou privés. 


\title{
Modeling of electrochemically generated bubbly flow under buoyancy-driven and forced convection
}

\author{
Jonathan Schillings ${ }^{1,2}$, Olivier Doche ${ }^{1,2}$, Jonathan Deseure ${ }^{1,2}$ \\ ${ }^{1}$ Univ. Grenoble Alpes, LEPMI, F-38000 Grenoble, France \\ ${ }^{2}$ CNRS, LEPMI, F-38000 Grenoble, France
}

\begin{abstract}
This work is devoted to the modeling of two phase flows arising in typical electrolysis devices. A numerical mixture model is used in order to resolve the two dimensional bubble plumes evolving along the electrodes. Plumes thickness sensitivity is studied for various parameters, such as bubble diameter, electrolyte viscosity, electrochemical cell geometry and current density. Using thermal buoyancy driven flow analogy, a dimensionless Rayleigh-like number $\boldsymbol{R} \boldsymbol{\alpha}_{f, \boldsymbol{e}}$ is defined to predict the behavior of the wallbounded gas convection between two vertical facing electrodes. Different bubbles dispersion mechanisms are observed depending on two-phase flow dynamics and physical properties of the mixture. The effect of forced convection in the channel is also investigated. A scaling law for plume thickness evolution for a large range of Prandtl-equivalent numbervalues is proposed. These results show that the bubble plume can be efficiently controlled by an imposed electrolyte velocity.
\end{abstract}

\section{1 - Introduction}

The efficiency of industrial electrochemical processes strongly depends on mass transfer. For example, electrochemical reactions can be enhanced by pumped electrolyte flow, which increases mass transfer at the electrode surface. Alternatively, a flow can be induced by the electrochemical bubble production. Therefore, the cell efficiency of many electrochemical industrial processes is affected by bubble existence, such as chlorate process, where hydrogen bubbles are produced at the cathode [1]. In Hall-Héroult process for the production of aluminum [2], carbon monoxide and carbon dioxide are produced at the bottom of the anode. Hence, bath/metal interface is affected by gas bubbles. Concerning zinc-air fuel cells, the performance is determined by active surface area, of which morphological modifications during charge and discharge are impacted by convection, among other mechanisms [3]. Furthermore in the energy field, Zeng and Zhang [4] showed that the additional resistance arising from partial coverage of the electrodes by the bubbles was critical in alkaline water electrolysis efficiency. A better understanding of bubble behavior would provide a scientific guidance to minimize this resistance and contribute to the development of hydrogen production. Generally, at gas-evolving electrodes, the actual distribution of current density differs from its nominal value. According to Vogt [5], empirical 
descriptions may be used in order to describe the gas evolving electrodes but are restricted to some bubble coverage ranges and particular operating points.

Nagai et al. [6] have shown that the decrease of electrolysis efficiency occurs by the increase of void fraction between electrodes. Due to the coupling between the electrochemistry and the two-phase flow, process performances are dependent on numerous experimental parameters such as electrode and reactor geometries, current density, electrochemical kinetics and electrolyte concentration. Even though the combined influence of all these factors might be complex to predict, the fact remains that optimum operating conditions can be found from experimental results together with a physical model of void fraction evolution. Furthermore, extrapolating results from laboratory to pilot and industrial scale requires careful consideration. Dimensionless parameters and correlations can be used to design and characterize industrial electrochemical cell, as for example, in the case of filter-press reactors [7].

However, in many industrial electrochemical applications (e.g. chlorate process, waste treatment, redox flow battery and electrolysis) there is a lack of practical tools to characterize, optimize and scale-up reactors.

The purpose of this paper is to describe how the mechanisms of bubble dispersion affect the global flow by means of a mixture (also called drift-flux) model. While an Eulerian-Eulerian two-fluid model (2FM) [8] or an Eulerian-Lagrangian liquid-bubble representation [9] would presumably be both more rigorous in the description of the dispersed phase, they would induce higher numerical costs. The mixture modelsimpler in its formulation and resolution - is well suited for the present study due to its good efficiency in the case of unidirectional flows and small bubbles [10]. A new boundary layer model is derived from this mixture formulation and with it, a Rayleigh-like and a Prandtl-like dimensionless numbers to characterize bubble-driven convection and plume behavior. It is worth mentioning that the analogy between two-phase flows and heat transfercan be found in the literature by using for instance the Rayleigh-Benard instability analogy [11-13] or nucleate boiling heat transfer properties for describing mass transfer at the electrodes [14]. 


\section{2- Model description}

\section{1- General description}

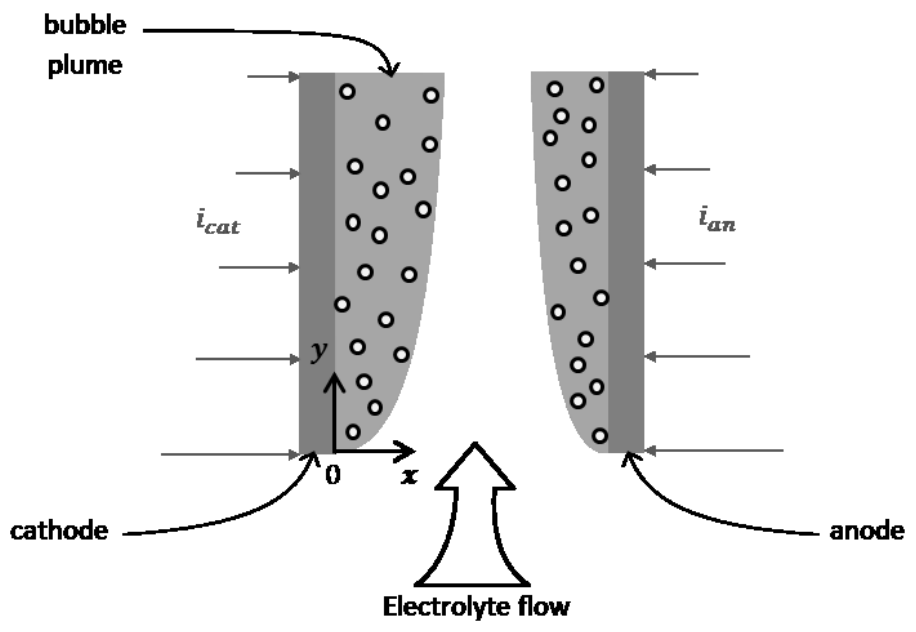

Fig. 1 : Schematic representation of the electrochemicallygenerated bubbly flow.

Fig. 1 shows an illustration of a standard electrolysis set-up and represents the configuration of the model. The facing electrodes are vertical plates with infinite depth (2D approximation). $\boldsymbol{x}$ and $\boldsymbol{y}$ stand respectively for the horizontal and vertical directions. The cathode is located at $\boldsymbol{x}=\mathbf{0}$, the anode at $\boldsymbol{x}=$ $\boldsymbol{2} \boldsymbol{e}$ and $\boldsymbol{y}=\mathbf{0}$ is set at the entrance of the channel. The electroactive length is noted $\boldsymbol{L}$ and the inter-plate gap is then $2 \boldsymbol{e}$. Generated bubbles evolve in plumes along the walls. The electrolyte flow can either be forced into the channel or be induced by buoyancy forces arising from the bubbles creation.

\section{2- Mixture model}

In the present study, the two-phase flow dynamics is modeled by the mixture formulation as expressed by Ishii [15]. The quantities $\overrightarrow{\boldsymbol{v}_{\boldsymbol{D}}}$ and $\overrightarrow{\boldsymbol{v}_{\boldsymbol{c}}}$ are respectively the dispersed and the continuous phase velocities. $\overrightarrow{\boldsymbol{q}}$ and $\overrightarrow{\boldsymbol{U}}$ are respectively the mass-and volume-averaged (also called superficial) velocity fields of the mixture and $\boldsymbol{\rho}=(\mathbf{1}-\boldsymbol{\alpha}) \boldsymbol{\rho}_{C}+\boldsymbol{\alpha} \boldsymbol{\rho}_{D}$, its density. Then, the following set of relations is obtained:

$$
\begin{gathered}
\overrightarrow{U_{D}}=\alpha \overrightarrow{v_{D}}, \\
\overrightarrow{U_{C}}=(1-\alpha) \overrightarrow{v_{C}}, \\
\vec{U}=\overrightarrow{U_{D}}+\overrightarrow{U_{C}}, \\
\rho \vec{q}=\rho_{C} \overrightarrow{U_{C}}+\rho_{D} \overrightarrow{U_{D}}
\end{gathered}
$$

The subscripts $C$ and $D$ stand respectively for the continuous and dispersed phase. Let $\overrightarrow{\boldsymbol{U}_{\boldsymbol{r}}}$, the relative volume flux of the dispersed phase, be related to $\overrightarrow{\boldsymbol{U}}$ and $\overrightarrow{\boldsymbol{q}}$ by the following equations: 


$$
\overrightarrow{U_{r}}=\overrightarrow{U_{D}}-\alpha \vec{U}
$$

Due to the incompressibility condition both density of the continuous and dispersed phase are assumed to be constant. In addition, $\rho_{D} \ll \rho_{c}$ and no phase change is considered in this work.

Two-phase flow is governed by the following set of equations:

$$
\begin{gathered}
\vec{\nabla} \cdot(\vec{U})=\vec{\nabla} \cdot\left(\overrightarrow{U_{D}}\right)+\vec{\nabla} \cdot\left(\overrightarrow{U_{C}}\right)=0 \\
\vec{\nabla} \cdot\left(\overrightarrow{U_{D}}\right)=\vec{\nabla} \cdot\left(\overrightarrow{U_{r}}\right)+\vec{\nabla} \cdot(\alpha \vec{U})=0 \\
\rho \vec{q} \cdot \nabla \vec{q}=-\vec{\nabla} P+\vec{\nabla} \cdot\left[\mu\left(\nabla \vec{q}+\nabla \vec{q}^{T}\right)-\frac{2}{3} \mu(\vec{\nabla} \cdot \vec{q}) I\right]-\rho_{c} \alpha \vec{g}
\end{gathered}
$$

Equations (2.3), (2.4) and (2.5) stand respectively for the mixture volume conservation, disperse d-phase volume conservation and mixture momentum conservation, where $\boldsymbol{P}=\boldsymbol{p}+\boldsymbol{\rho}_{\boldsymbol{C}} \boldsymbol{g} \boldsymbol{y}$ is the reduced pressure and $\boldsymbol{\mu}=\frac{\boldsymbol{\mu}_{\boldsymbol{C}}}{(\mathbf{1}-\boldsymbol{\alpha})}$ is an empirical expression of mixture dynamic viscosity, given by Ishii and Zuber [16]. The last term in equation (2.5) stands for the buoyancy forces. The thermal-driven buoyancy due to heat generation/absorption during the electrochemical process is negligible compared to the bubbledriven buoyancy. To illustrate this, a void fraction of $1 \%$ gives rise to the same buoyancy forces as an increase of $50^{\circ} \mathrm{C}$ in water. Furthermore, because of the strong mixing induced by the two-phaseflow near the electrode surfaces, there is no significant ion concentration gradient.

The description of bubbles relative motion is adapted from an empirical model of sedimenting spherical rigid particles in laminar flow over an inclined surface, thus neglecting the phenomena of bubble deformation, internal circulation, adherence on the walls and bubble coalescence and break-off. These assumptions are not unreasonable as it is commonly admitted that hydrogen bubbles in alkaline electrolyte are sufficiently small to remain spherical and do not coalesce easily [17]. Although oxygen bubbles are significantly bigger than hydrogen ones, this remains a good approximation to represent the main behaviors of bubble plumes. This simplification was first formulated in the work of Dahlkild [18] for the boundary layer model of an isolated gas-evolving electrode, and then the formulation of the relative velocity was completed by Wedin and Dahlkild [19] for the mixture model. The terms of this formulation were adapted from various studies [20-23]. It is written as coupled interactions:

$$
\begin{gathered}
\overrightarrow{U_{r}}=\overrightarrow{U_{\text {Stokes }}}+\overrightarrow{U_{\text {Saff }}}+\overrightarrow{U_{\text {Hdıff }}}+\overrightarrow{U_{\text {Sdlff }}}+\overrightarrow{U_{m i g}} \\
\overrightarrow{U_{\text {Stokes }}}=\alpha f(\alpha) v_{\text {Stokes }} \overrightarrow{e_{y}} \\
\overrightarrow{U_{\text {Saff }}}=-\alpha f(\alpha) v_{\text {Stokes }} \operatorname{sgn}(\dot{\gamma}) \frac{6.46}{6 \pi} \sqrt{\frac{r_{b}{ }^{2}|\dot{\gamma}|}{v_{C}} \overrightarrow{e_{x}}} \\
\overrightarrow{U_{\text {Hdıff }}}=-r_{b} v_{\text {Stokes }} f(\alpha) D \vec{\nabla} \alpha \\
\overrightarrow{U_{\text {Sdıff }}}=-r_{b}{ }^{2}|\dot{\gamma}| \beta(\alpha) \vec{\nabla} \alpha
\end{gathered}
$$




$$
\overrightarrow{U_{S m i g}}=-r_{b}{ }^{2}|\dot{\gamma}| \frac{\kappa(\alpha)}{\tau} \vec{\nabla} \tau
$$

The first term $\overrightarrow{\boldsymbol{U}_{\text {Stokes }}}$ is the Stokes' flux which results from a balance between buoyancyand drag forces. $v_{\text {Stokes }}=\frac{\mathbf{2 g r _ { b }} \mathbf{2}^{2}}{\mathbf{9} v_{C}}$, the Stokes velocity, is the terminal rising velocity of a particle of radius $\boldsymbol{r}_{\boldsymbol{b}}$ in a liquid of cinematic viscosity $\boldsymbol{v}_{C}$ and $f(\alpha)=(\mathbf{1}-\alpha)^{\mathbf{5}}$ is the hindering function, formulated by Nicolai et al. [20], that stands for the modification of viscosity and density in side the plume.

The second term $\overrightarrow{\boldsymbol{U}_{\boldsymbol{S a f f}}}$ is a lift flux caused by rotation of particles in a sheared flow, and is derived from Saffman's lift velocity [21]. $\dot{\gamma}=\frac{\partial \boldsymbol{q}_{\boldsymbol{y}}}{\partial x}$ is the main shear rate term for a flow between vertical pates. Lift forces tend to push rising bubbles towards the lowest velocities.

The third term $\overrightarrow{\boldsymbol{U}_{\boldsymbol{H} \boldsymbol{d} \boldsymbol{l} f \boldsymbol{f}}}$ is the hydrodynamic self-diffusion, which describes the irregular path of bubbles in a plume, resulting in a general diffusive behavior. $\boldsymbol{D}=\left(\begin{array}{cc}\boldsymbol{D}_{\perp} & \mathbf{0} \\ \mathbf{0} & \boldsymbol{D}_{\|}\end{array}\right)$is a non-isotropic dimensionless dispersion coefficient, measured by Nicolai et al. [20], which values $\boldsymbol{D}_{\perp} \sim \mathbf{1}$ and $\boldsymbol{D}_{\|} \sim \mathbf{8}$ are approximately constant and independent of $\boldsymbol{\alpha}$.

Finally, the last terms are due to an increase of collision frequency in a sheared flow, proportional to $\boldsymbol{\alpha}|\dot{\gamma}|$, as reported in the literature [22,23]. $\overrightarrow{\boldsymbol{U}_{\boldsymbol{S d \boldsymbol { l }} \boldsymbol{f} \boldsymbol{f}}}$ is the shear-induced diffusion, generated by bubble concentration gradient in a constant shear and $\overrightarrow{\boldsymbol{U}_{\boldsymbol{S m i g}}}$ is the shear-induced migration for non-uniform shear stresses. $\boldsymbol{\tau}=\boldsymbol{\mu} \frac{\partial q_{y}}{\partial x}$ is the preponderant term of shear stress tensor, and $\boldsymbol{\beta}(\boldsymbol{\alpha})=\frac{\mathbf{1}}{\mathbf{3}} \boldsymbol{\alpha}^{\mathbf{2}}(\mathbf{1}+$ $0.5 e^{8.8 \alpha}$ ) and $\boldsymbol{\kappa}(\alpha)=0.6 \alpha^{2}$ are non-dimensional coefficients.

\section{3 - Electrochemical reactions}

For the standard case of alkaline water electrolysis, the following half-reactions take place respectively at the anode and at the cathode:

$$
\begin{gathered}
2 \mathrm{OH}^{-} \rightarrow \frac{1}{2} \mathrm{O}_{2}+\mathrm{H}_{2} \mathrm{O}+2 e^{-} \\
2 \mathrm{H}_{2} \mathrm{O}+2 e^{-} \rightarrow \mathrm{H}_{2}+2 \mathrm{OH}^{-}
\end{gathered}
$$

Gas fluxes leaving the electrodes surface are linked to the electrical current density by means of Faraday's law, written in (2.14). The main considerations are that hydrogen and oxygen are ideal gases, the Faradaic yield is total and the products are generated in gaseous state.

$$
U_{H 2}=\frac{1}{2} \frac{R T i_{a v}}{p F}, \quad U_{O 2}=\frac{1}{4} \frac{R T i_{a v}}{p F}
$$

where $\boldsymbol{R}$ is the universal gas constant, $\boldsymbol{T}$ the operating temperature, $\boldsymbol{p}$ the operating pressure, $\boldsymbol{F}$ is the Faraday constant and $\boldsymbol{i}_{\boldsymbol{a} \boldsymbol{v}}$ is the average current density. 

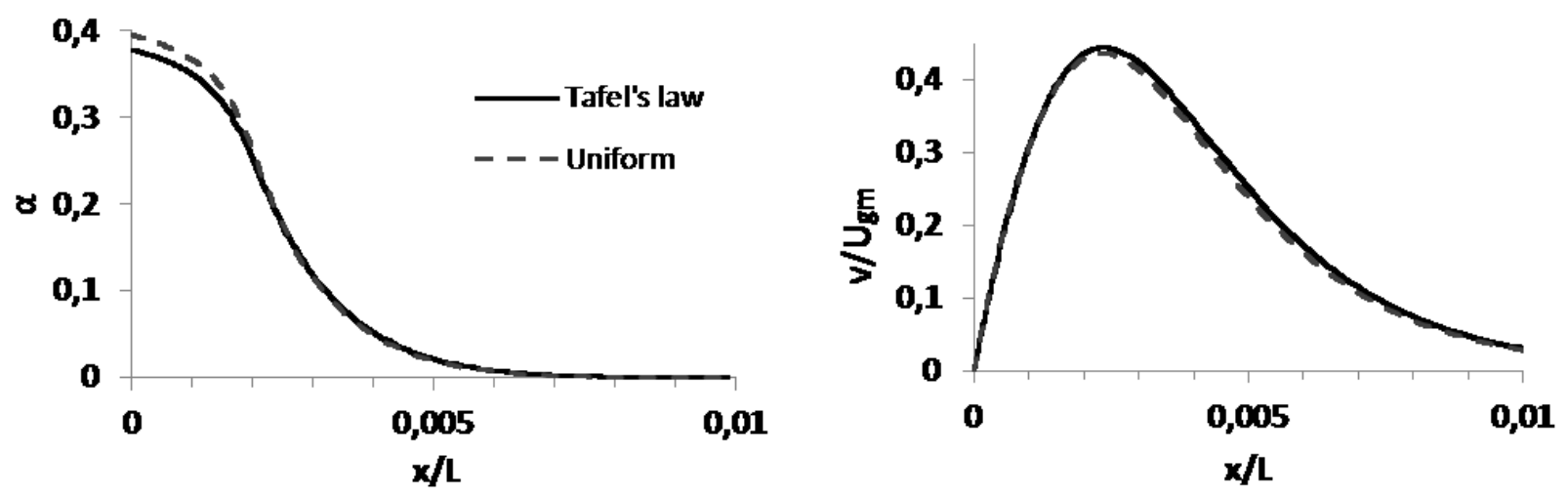

Fig. 2 : Void fraction (left) and velocity (right) evolution in the direction perpendicular to cathode surface, calculated at the outlet of the cathode for a non-uniform (full line) and an averaged uniform (dashed line) current distribution.

Fig. 2 exhibits the simulated results for a vertical cathode of height $\boldsymbol{L}$ exposed to semi-infinite electrolyte. A first case of simulations are performed with electrical current distribution described using a modified Tafel's law [18]:

$$
i=i_{0}(1-\alpha) e^{\left(-\frac{F}{2 R T} \eta\right)}
$$

where $\boldsymbol{\eta}$ is the overpotential set to be equal to the opposite of the electrolyte potential $(\boldsymbol{\phi})$ at the interface with the electrode surface. Due to the large distance assumptions (one electrode surrounded by semiinfinite electrolyte) and infinite dilut ed species consideration, the charge balance (electronic and ionic) can be simplified as follow:

$\boldsymbol{\nabla} . \boldsymbol{i}_{\boldsymbol{x}}=\mathbf{0}$ and $\boldsymbol{i}_{\boldsymbol{x}}=\boldsymbol{\sigma} \overrightarrow{\boldsymbol{\nabla} \boldsymbol{\phi}}$

The second case considers an equivalent but spatially averaged (i.e. uniform) current evolution along the electrodes. Fig. 2 (left) displays void fraction evolution perpendicular to cathode surface, at $\boldsymbol{y}=$ $\boldsymbol{L}$ and Fig. 2 (right) shows the dimensionless axial velocity. It can be clearly observed that the two-phase flow behavior is not significantly affected by a non-uniform current distribution along the electrodes. The plumes thickness at the outlet - which is the principal result when one aims to avoid mixing of facing bubble plumes - is almost similar in both cases (averaged and non-uniform current density). This is an important fact since it simplifies drastically the numerical resolution by avoiding any iterative procedure dedicated to the electrokinetics coupling. The simulation time of an isolated electrode could, for example, be divided by a factor up to 8 in some cases, and the gain might be higher for two electrodes undergoing different electrochemical kinetics. Since process performances (cell tension, ohmic losses) are not of prime importance, the uniform current approximation will be used in the rest of the study.

The 2D finite elements resolution with Comsol Multiphysics was validated by direct comparison with the numerical results of Dahlkild [18] for an isolated electrode. In order to validate two-electrode cases, the results of simulations were compared to the experimental results of Boissonneau and Byrne [1], as presented in the following section. 


\section{4 - Model validation with a free bubble-induced convection case}

Simulations results are compared to the experimental data of Boissonneau and Byrne [1]. In their work, a water electrolysis was studied inside a vertical channel of dimensions $3 \mathrm{~L}=120 \mathrm{~mm}, \mathbf{2} \mathrm{e}=\mathbf{3 m m}$ and $\boldsymbol{W}=\mathbf{3 0} \mathbf{m m}$ (depth). In their experimental apparatus, the initial length of the channel was electrochemically inactive in order to develop the flow without any perturbation from the dispersed phase. Setting the entrance of the channel at $\boldsymbol{y}=\mathbf{0}$, the channel walls were free of bubble generation along $\boldsymbol{y} \in$ $[\mathbf{0}, \boldsymbol{L}]$. The lateral channel walls were electroactive for $\boldsymbol{y} \in[\boldsymbol{L}, \mathbf{2} \boldsymbol{L}]$ and once again inactive for $\boldsymbol{y} \in[\mathbf{2} \boldsymbol{L}, \mathbf{3} \boldsymbol{L}]$ to avoid any effect of the outlet on the inner profiles. The channel was immersed into a bath which could be considered as infinite and quiescent. The electrolyte was an aqueous solution of $\mathrm{Na}_{2} \mathrm{SO}_{4}$ concentrated at $50 \mathrm{~g} / \mathrm{L}$. The electrolyzer was operated at room temperature, atmospheric pressure and under different current density values $\left(500,1000\right.$ and $\left.2000 \mathrm{~A} / \mathrm{m}^{2}\right)$. They measured bubbles diameters at the bottom and top of their channel for each current density and found values between 30 and $80 \mu \mathrm{m}$, which is small enough to consider them as rigid spheres. The corresponding properties usedin the simulations are listed in Table 1 below:

Table 1 : Physical properties corresponding to the operating conditions in the work of Boissonneau and Byrne [1].

\begin{tabular}{|c|c|c|}
\hline$T\left({ }^{\circ} \mathrm{C}\right)$ & & 25 \\
\hline$P($ atm $)$ & & 1 \\
\hline$\rho_{C}\left(k g \cdot m^{-3}\right)[24]$ & & 1040 \\
\hline$v_{C}\left(m^{2} \cdot s^{-1}\right)[24]$ & & 0.997 \\
\hline \multirow[t]{2}{*}{$r_{b}(\mu m)$ at $500 \mathrm{~A} / \mathrm{m}^{2}$} & $y \in[L, 2 L]$ & $y \in[2 L, 3 L]$ \\
\hline & $15+15 \frac{y-L}{L}$ & 30 \\
\hline \multirow[t]{2}{*}{$r_{b}(\mu m)$ at $1000 \mathrm{~A} / \mathrm{m}^{2}$} & $y \in[L, 2 L]$ & $y \in[2 L, 3 L]$ \\
\hline & $22+10 \frac{y-L}{L}$ & 32 \\
\hline \multirow[t]{2}{*}{$r_{b}(\mu m)$ at $2000 \mathrm{~A} / \mathrm{m}^{2}$} & $y \in[L, 2 L]$ & $y \in[2 L, 3 L]$ \\
\hline & $30+10 \frac{y-L}{L}$ & 40 \\
\hline
\end{tabular}

In every cases, the resulting liquid flow was established before entering the electrodes, according to the laminar entrance length $L_{i n}=0.04 R e_{D_{H}} e$ [25], where $\operatorname{Re}_{D_{H}}=\frac{v_{\text {mean }} D_{H}}{v_{C}}, \boldsymbol{v}_{\text {mean }}$ is the measured mean velocity at the inlet of the channel, $\boldsymbol{e}$ is the semi-inter-electrode gap and $\boldsymbol{D}_{\boldsymbol{H}}=\mathbf{4} \boldsymbol{e}$ is the hydraulic diameter in a reasonable 2D approximation of the channel (infinite depth). The channel Reynolds number $\boldsymbol{R} \boldsymbol{e}_{\boldsymbol{D}_{\boldsymbol{H}}}$ remained under 600, suggesting that the flow stays laminar in each experiment, which is confirmed by the Poiseuille flow profile observed at the bottom of the electrodes as shown on Fig. 3.

According to the experiments, simulations were run with the following boundary conditions:

$$
\boldsymbol{y}=\mathbf{0}
$$

$$
\left\{\begin{array}{c}
P_{\text {in }}=-\frac{1}{2} \rho_{C} q_{y, \text { mean }}{ }^{2} \\
\alpha=0
\end{array}\right.
$$




$$
\begin{aligned}
& y=3 L \\
& P_{\text {out }}=0 \\
& \boldsymbol{x}=\mathbf{0} \quad\left\{\begin{array}{r}
\boldsymbol{q}_{\boldsymbol{x}}=\mathbf{0} \\
\boldsymbol{q}_{\boldsymbol{y}}=\mathbf{0} \\
\boldsymbol{U}_{\boldsymbol{r} \boldsymbol{x}}=\frac{\boldsymbol{U}_{\boldsymbol{H} 2}}{\mathbf{1}-\boldsymbol{\alpha}}, \text { for } y \in[L ; 2 L] \text { and } \boldsymbol{U}_{\boldsymbol{r} \boldsymbol{x}}=\mathbf{0} \text { elsewise }
\end{array}\right. \\
& \boldsymbol{x}=\boldsymbol{H}\left\{\begin{array}{r}
\boldsymbol{q}_{\boldsymbol{x}}=\mathbf{0} \\
\boldsymbol{q}_{\boldsymbol{y}}=\mathbf{0} \\
\boldsymbol{U}_{\boldsymbol{r} \boldsymbol{x}}=-\frac{\boldsymbol{U}_{\boldsymbol{O} 2}}{\mathbf{1}-\boldsymbol{\alpha}}, \text { for } y \in[L ; 2 L] \text { and } \boldsymbol{U}_{\boldsymbol{r} \boldsymbol{x}}=\mathbf{0} \text { elsewise }
\end{array}\right.
\end{aligned}
$$

The channel is immersed in an infinite quiescent liquid; this can be modeled by pressure conditions at both the inlet and the outlet. The reduced pressure at the outlet is set to 0 , while the inlet value is smaller due to the depression caused by the acceleration of the liquid. By means of an iterative procedure, the inlet pressure is thus adjusted to $-\frac{1}{\mathbf{2}} \boldsymbol{\rho}_{\boldsymbol{C}} \boldsymbol{q}_{\boldsymbol{y}, \text { mean }}{ }^{\mathbf{2}}$, where $\boldsymbol{q}_{\boldsymbol{y} \text {,mean }}$ is the averaged inlet velocity. This type of approximation is also used in thermal natural convection simulations (see Dalbert et al. [26]). Eqs (2.18) (a-c) and (2.19) (a-c) represent a wall condition for the condensed phase and inward uniform fluxes normal to the electrodes for the dispersed phase.

Numerical results exhibited in Fig. 3 are consistent with the velocity measurements of Boissonneau \& Byrne [1] taken at different heights, although the total experimental flow rate (including wall gas injection) is not conserved through the channel. Simulation results show clearly that the mixture is accelerated near the walls due to buoyancy forces, dragging the liquid from the center to the wall. Since the volume production of hydrogen (at $x=0 \mathrm{~mm}$ ) is twice that of the oxygen $(x=3 \mathrm{~mm})$, the velocity is significantly higher at the cathode (i.e the left electrode). This difference is somehow less remarkable on the experimental results but is still noticeable. 

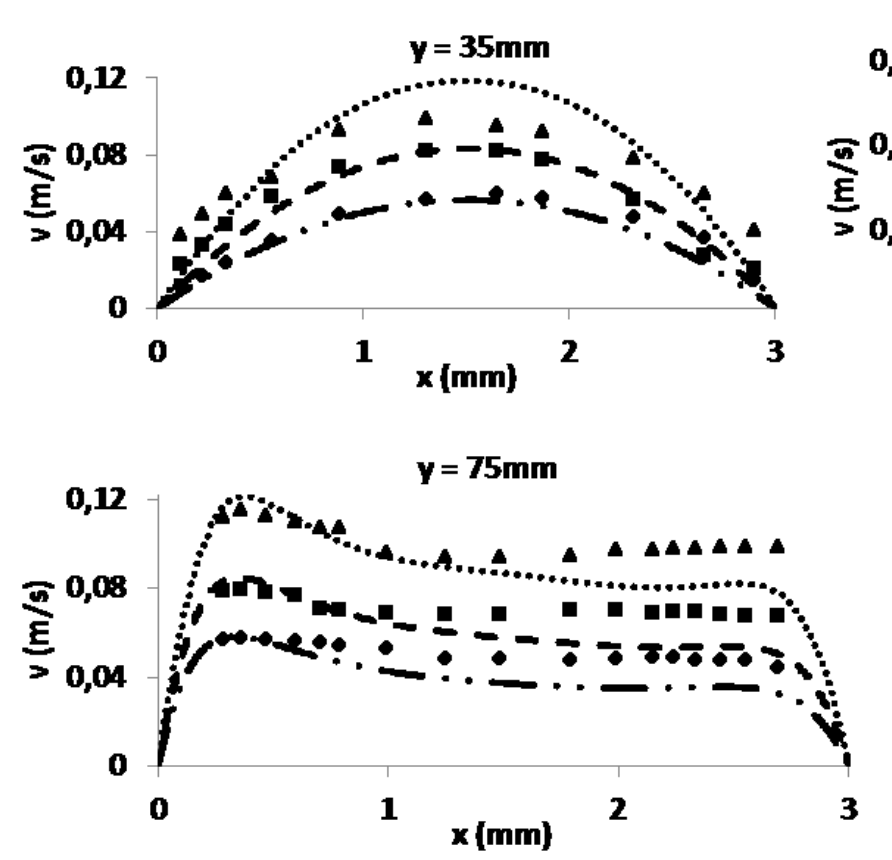
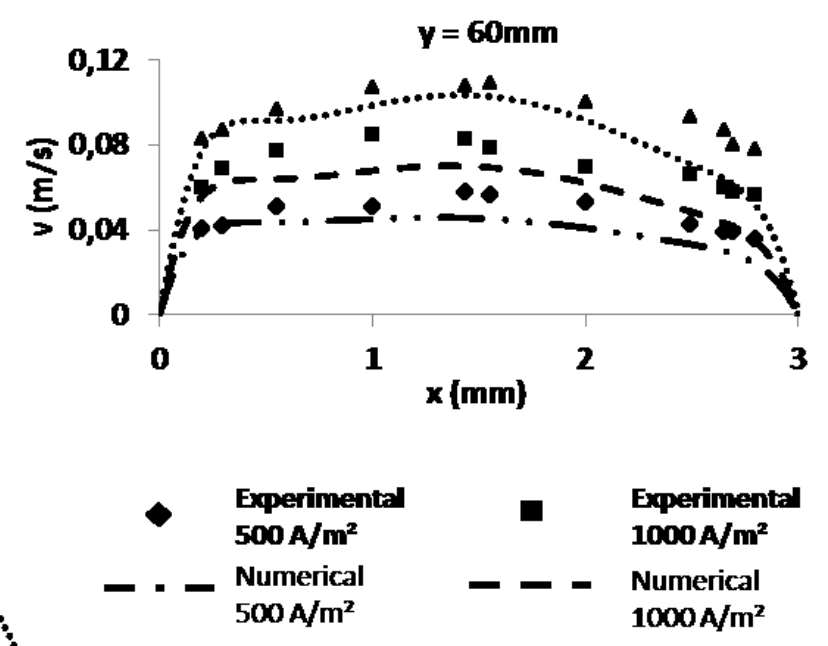

Experimental 2000A/m $\mathbf{m}^{2}$ Numerical $2000 \mathrm{~A} / \mathrm{m}^{2}$

Fig. 3 : Experimentally measured velocity profiles [1] versus simulation results.

\section{3- Plume behavior}

\section{1 - Thermal analogy}

In the case of vertical gas evolving electrodes, analogy with natural convection between vertical heated plates is almost straightforward. A bubble plume started at the surface of an electrode can be described as a boundary layer of size $\boldsymbol{\delta}_{\alpha}$ in a similar way of its thermal $\boldsymbol{\delta}_{T}$ counterpart. Thorough descriptions of thermal boundary layers can be found in the work of Bejan [25]. The aim is to roughly segregate the inner "near wall" region (i.e. $<\boldsymbol{\delta}_{\boldsymbol{\alpha}}$ ) where buoyancy forces balance the brake effect of viscous forces at the wall and the outer region (i.e. $>\boldsymbol{\delta}_{\boldsymbol{\alpha}}$ ) where inertia prevails on buoyancy forces. Buoyancy forces drive the vertical flow in the channel and the scalar $\boldsymbol{\alpha}$ follows a diffusion-convection law similar to, but somehow more complex than, the conservation of energy for temperature. Combining (2.3) and (2.4):

$$
U_{x} \frac{\partial \alpha}{\partial x}+U_{y} \frac{\partial \alpha}{\partial y}=\frac{\partial U_{r x}}{\partial x}+\frac{\partial U_{r y}}{\partial y}
$$

We consider a simple 2D geometry constituted of two vertical electrodes of height $\boldsymbol{L}$ separated by a distance $2 \boldsymbol{e}$. Each electrode generates a mean gas flux $\boldsymbol{U}_{\boldsymbol{g}}$ uniformly distributed on its surface. A scale analysis allows defining the typical values of the problem. Let the following orders of magnitude in the inner region of the boundary layer be chosen as: $\boldsymbol{\alpha} \sim \boldsymbol{A}, \boldsymbol{q}_{\boldsymbol{y}} \sim \boldsymbol{U}_{\boldsymbol{y}} \sim \boldsymbol{V}, \boldsymbol{q}_{\boldsymbol{x}} \sim \boldsymbol{U}_{\boldsymbol{x}} \sim \boldsymbol{W}, \boldsymbol{y} \sim \boldsymbol{L}$ and $\boldsymbol{x} \sim \boldsymbol{\delta}_{\boldsymbol{\alpha}}$. Volume conservation in (2.3) leads to $\frac{V}{L} \sim \frac{W}{\delta_{\alpha}}$, so that the scale for the two convective terms on the lefthand side of Eq. (3.1) is $\frac{V A}{L}$. The boundary layer model also implies that $\frac{\partial}{\partial x} \sim \frac{1}{\delta_{\alpha}} \gg \frac{1}{L} \sim \frac{\partial}{\partial y}$ meaning that the 
vertical components of dispersive terms (2.9), (2.10) and (2.11) are negligible, and that $\frac{\partial U_{\text {Saff }}}{\partial x} \gg \frac{\partial U_{\text {Stokes }}}{\partial y}$. The horizontal components of (2.10) and (2.11) both scale with $\frac{r_{b}{ }^{2}|\dot{\gamma}|}{\delta_{\alpha}}$. Therefore, the balance between convection and dispersion as described in (3.1) can be simplified to (3.2):

$$
\frac{V A}{L} \sim \frac{U_{\text {Saff }}}{\delta_{\alpha}}, \frac{U_{\text {Hdiff }}}{\delta_{\alpha}}, \frac{U_{\text {Sdiff }}}{\delta_{\alpha}}
$$

Hence, (3.2) can be written in the form of (3.3):

$$
\frac{V A}{L} \sim K_{\alpha} \frac{A}{\delta_{\alpha}{ }^{2}}
$$

where $\boldsymbol{K}_{\boldsymbol{\alpha}}$ is an equivalent dispersion coefficient analogous to thermal diffusion coefficient. The Saffman's lift, hydrodynamic self-diffusion and shear-induced diffusion terms contribute to $\boldsymbol{K}_{\boldsymbol{\alpha}}$. Their respective magnitudes depend on flow conditions as shown in subsections 3.2 and 3.3.

Inside the channel, the balance between viscous and buoyancy forces can be expressed as:

$$
v_{C} \frac{V}{e^{2}} \sim g\left(A \frac{\delta_{\alpha}}{e}\right)
$$

where $\boldsymbol{A} \frac{\boldsymbol{\delta}_{\alpha}}{\boldsymbol{e}}$ is the void fraction averaged over the whole fluid domain.

The mean gas flux injection can be approximated by:

$$
U_{g} \sim K_{\alpha} \frac{A}{\delta_{\alpha}}
$$

A Prandtl-like number can be defined as:

$$
\operatorname{Pr}_{\alpha}=\frac{v_{C}}{K_{\alpha}}
$$

$\boldsymbol{P} \boldsymbol{r}_{\boldsymbol{\alpha}}$ compares the momentum diffusion to the void fraction dispersion. For highervalues of $\boldsymbol{P} \boldsymbol{r}_{\boldsymbol{\alpha}}$, slenderer bubble plumes are observed.

Fig. 4 shows a scheme of velocity and void fraction profiles for $\boldsymbol{P r}_{\alpha} \gg \mathbf{1}$ and $\boldsymbol{P r}_{\boldsymbol{\alpha}} \ll \mathbf{1}$. Both cases will be described separately in the following sections. Notice that for sake of clarity, the oute $r$ boundary velocity was set to zero on Fig. 4. This is not true in the real case for which the core (i.e. pure liquid) region velocity should evolve along the electrode height. 

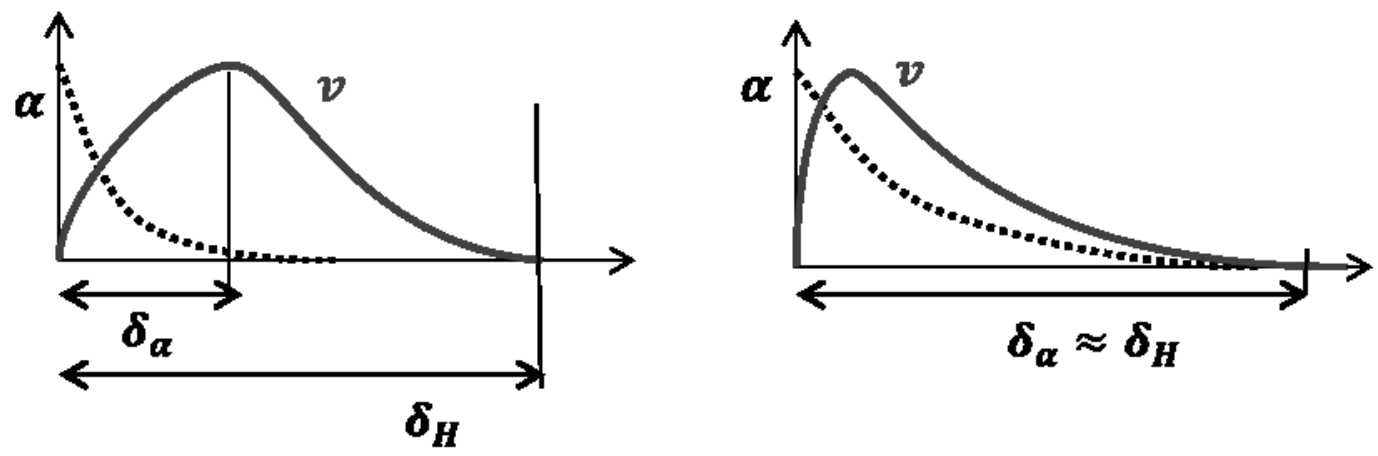

Fig. 4 : Typical velocity (full line) and void fraction (dotted line) distributions in the vicinity of electrodes, for high (left) a nd low (right) Pra values [25].

\section{$3.2-P r_{\alpha} \gg 1$}

In this case, as depicted on the left side of Fig. 4, the plume thickness is very small compared to the hydrodynamic boundary layer thickness. The mixture is accelerated in the presence of bubbles and then the velocity decreases until reaching the distance $\boldsymbol{\delta}_{\boldsymbol{H}}$. These kinds of plume are highly concentrated in bubbles and undergo a strong positive shear. The three terms influent in bubble relative motion are the hydrodynamic self-diffusion, the shear-induced diffusion and Saffman's lift force. Two asymptotical cases exist. For current densities sufficiently low to ensure that $\boldsymbol{\beta}(\boldsymbol{\alpha})$, and thus $\boldsymbol{U}_{\text {Sdiff }}$, are negligible, lift forces and hydrodynamic diffusion prevail so that $K_{\alpha} \sim v_{\text {Stokes }} \boldsymbol{r}_{b}\left(1-\sqrt{\frac{|\bar{\gamma}|}{v_{C}}} \delta_{\alpha}\right) \sim v_{\text {Stokes }} \boldsymbol{r}_{\boldsymbol{b}}\left(1-\sqrt{\frac{\boldsymbol{V}_{\text {max }} \boldsymbol{\delta}_{\alpha}}{\boldsymbol{v}_{C}}}\right)$. Where $\boldsymbol{V}_{\text {max }}$ is the maximum velocity, reached at $\boldsymbol{x} \approx \boldsymbol{\delta}_{\boldsymbol{\alpha}}$. Thus, it is seen that the equivalent diffusivity depends on a velocity and a boundary layer thickness. In order to define a practical equivalent Prandt/ number, we choose to set $\boldsymbol{K}_{\boldsymbol{\alpha}} \sim \boldsymbol{v}_{\text {Stokes }} \boldsymbol{r}_{\boldsymbol{b}}$. This choice is confirmed by numerical evaluation of both diffusive terms in our simulations showing that lift and hydrodynamic diffusion are equivalent in magnitude. It is then important to keep in mind that $\boldsymbol{P} \boldsymbol{r}_{\boldsymbol{\alpha}}$ can be used to define scaling laws and perform sensitivity analysis. However for the set-up of complete correlations, exhaustive studies are necessary. By combining (3.3), (3.4) and (3.5) both $\boldsymbol{A}$ and $\boldsymbol{V}$ can be eliminated and one obtains the relation $\frac{\delta_{\alpha}{ }^{4}}{e^{4}} \sim \frac{K_{\alpha}{ }^{2} L v_{c}}{g U_{g} e^{5}}$. Hence, the Rayleigh-like numbercan be defined as $R \alpha_{f, e}=\frac{v_{c} U_{g} e^{5}}{r_{b}{ }^{6} L g}$, leading to the scaling law relation:

$$
\frac{\delta_{\alpha}}{e} \sim\left(R \alpha_{f, e}\right)^{-1 / 4}
$$

$\boldsymbol{f}$ and $\boldsymbol{e}$ subscript signal that the dimensionless number $\boldsymbol{R} \boldsymbol{\alpha}_{\boldsymbol{f}, \boldsymbol{e}}$ is expressed for a uniform flux condition and a characteristic length $\boldsymbol{e}$. The relative plume thickness $\frac{\delta_{\alpha}}{\boldsymbol{e}}$ is a key parameter for useful applications of electrolysis. It measures the distance between the two facing void fraction boundary layer and then allows to evaluate the potential merging (with chemical recombination) of the plumes. 


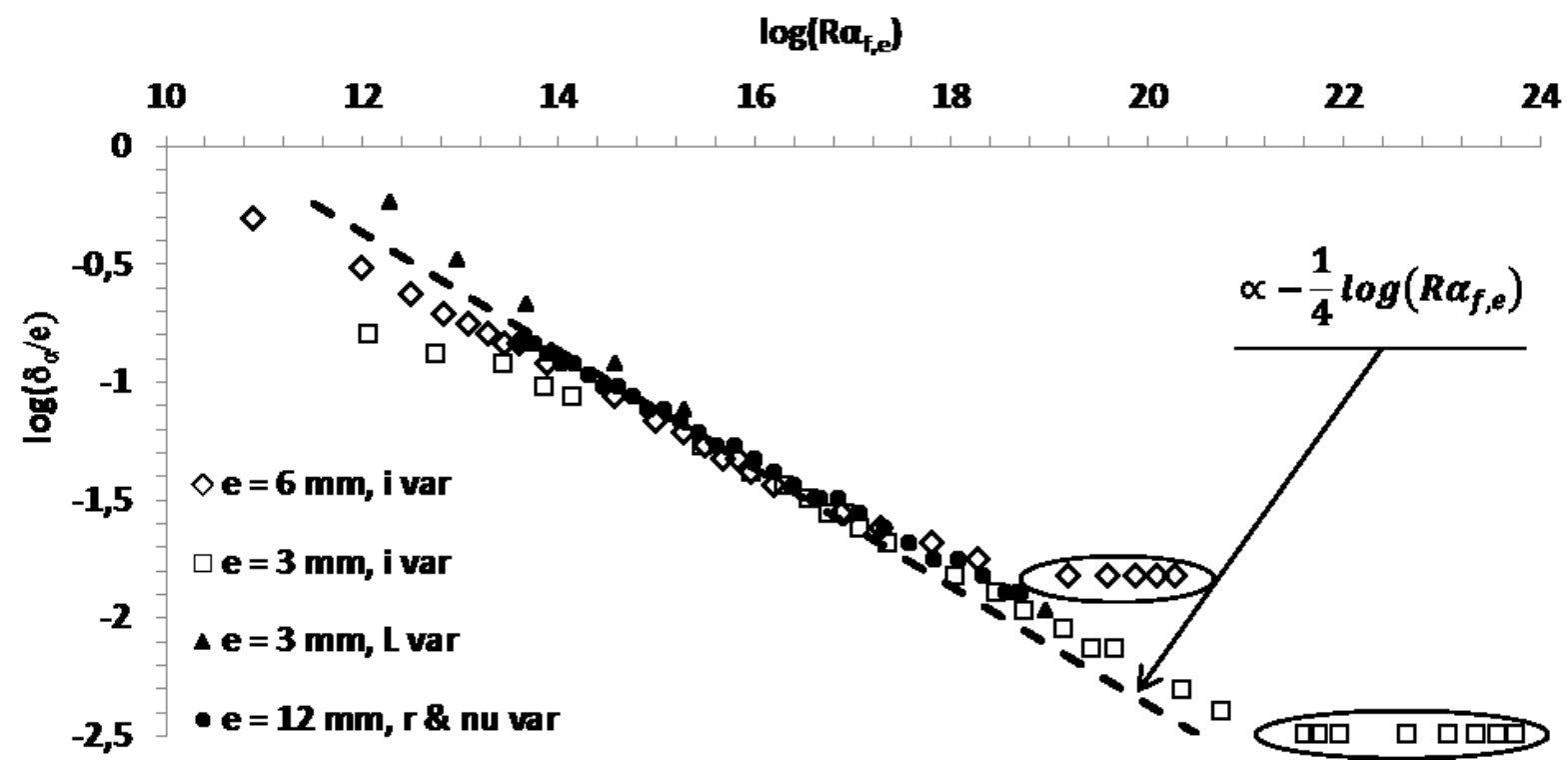

Fig. 5 : Relative plume thickness for a wide range of $R \alpha_{f, e}$. Sensitivity to current density was recorded at e $=3 \mathrm{~mm}$ (squares) and $6 \mathrm{~mm}$ (diamonds). Sensitivity to $r$ and $v_{\mathrm{C}}$ (circles) and to $L$ (triangles) are also displayed L. In every cases, $\operatorname{Pr}_{\alpha}>80$.

For sufficiently high current densities, shear rate and void fraction values at the electrode surface become extremely high. Thus, shear-induced diffusion prevails and $\boldsymbol{K}_{\boldsymbol{\alpha}} \sim \boldsymbol{r}_{\boldsymbol{b}}{ }^{2} \frac{\boldsymbol{V}}{\delta_{\alpha}}$. With this formulation, (3.3) becomes (3.8) and the relation for plume thickness shows only a dependency on geometrical parameters:

$$
\frac{\delta_{\alpha}}{e} \sim \frac{\left(r_{b}^{2} L\right)^{1 / 3}}{e}
$$

Fig. 5 reports results of simulations obtained for various values of $\boldsymbol{R} \boldsymbol{\alpha}_{f, \boldsymbol{e}}$ along with sensitivity analysis of principal parameters such as $\boldsymbol{e}, \boldsymbol{L}, \boldsymbol{i}_{\boldsymbol{a}}, \boldsymbol{v}_{\boldsymbol{c}}$ and $\boldsymbol{r}_{\boldsymbol{b}}$. It displays $\frac{\boldsymbol{\delta}_{\boldsymbol{\alpha}}}{\boldsymbol{e}}$ versus $\boldsymbol{R} \boldsymbol{\alpha}_{f, \boldsymbol{e}}$ plotted in log-scale at $\boldsymbol{P r}_{\boldsymbol{\alpha}}>\mathbf{8 0}$. The thickness of the void fraction boundary layer $\left(\boldsymbol{\delta}_{\alpha}\right)$ is measured at the top of the electrodes and is defined as the distance from the wall where void fractions is superior to $1 \%$ of its maximum value (located at the electrode surface in our configuration). In terms of power law, simulation results fit well with the evolution predicted by scale analysis. In a general manner, the value of $\log \left(\frac{\delta_{\alpha}}{e}\right)$ decreases almost linearly with $\log \left(\boldsymbol{R} \boldsymbol{\alpha}_{f, e}\right)$ and, thus, shows that the $-1 / 4$ logarithmic slope value of the Rayleigh equivalent number is quite representative on a large part of the tested range. However, the linearity is not perfect and, to the authors opinion, it could be explained by the flow-dependent nature of the dispersion coefficient $\boldsymbol{K}_{\boldsymbol{\alpha}}$. For high current densities (i.e. encircled squares and diamonds), the flow dynamic is mainly governed by shear-induced diffusion which overwhelms other diffusive effects. In this case, the ratio $\frac{\delta_{\alpha}}{e}$ is no more affected by $R \alpha_{f, e}$ as shown by previous analysis (eq. (3.8))

The results show that it is possible to predict (and control) the plume thickness variation by acting on different parameters, such as electrolyte viscosity or current density. This is also the first step of correlations build-up process depending on $\boldsymbol{R \alpha}_{f, \boldsymbol{e}}, \boldsymbol{P r}_{\boldsymbol{\alpha}}$ and geometrical configurations. Moreover, the 
gap $\boldsymbol{e}$ is of prime importance for the two-phase flow evolution along the electrodes because it is closely related to the pumping mechanism of the electrolyte arising from flow conservation. It is also important to notice that the most influent parameter is identified as the bubble size. Although it is generally not a tunable parameter in experiments, it has been shown $[17,27]$ that bubbles diameter can be controlled by the current density, salt concentration or pressure, or by adding surfactants in the solution.

\section{$3.3-P r_{\alpha} \ll 1$}

For low $\boldsymbol{P r}_{\boldsymbol{\alpha}}$ values, bubble plumes are typically less concentrated and thicker. Hence, shearinduced diffusion can be neglected. Fig. 4 shows, on the right-hand side, that void fraction dispersion can be governed either by a positive shear rate near the electrode, either by a negative one farther into the core of the flow where the velocity tends to decrease. Thus, the dispersion experiences a change in the resulting lift forces sign which makes difficult a consistent scale identification for $\boldsymbol{K}_{\boldsymbol{\alpha}}$.

Simulations results show a similar sensitivity to parameters such as $\boldsymbol{e}, \boldsymbol{L}, \boldsymbol{\nu}_{\boldsymbol{c}}$ and $\boldsymbol{r}_{\boldsymbol{b}}$ but with different magnitude than previously observed for high $\boldsymbol{P r}_{\boldsymbol{\alpha}}$. However, increasing the mean current value at the electrodes $\left(\boldsymbol{i}_{\boldsymbol{a}}\right)$ leads to a more complex behavior. Fig. 6 displays the dimensionless void fraction boundary layer thickness $\frac{\delta_{\alpha}}{e}$ versus $\boldsymbol{i}_{\boldsymbol{a} v}$ at $\boldsymbol{P} \boldsymbol{r}_{\boldsymbol{\alpha}}=\mathbf{0 . 0 7}$. In the first place, due to strong convection effect, increasing the current density tends to reduce $\boldsymbol{\delta}_{\boldsymbol{\alpha}}$. A further increase of current density leads to a higher maximum velocity and higher negative shear rates. Thus, the effective coefficient $\boldsymbol{K}_{\boldsymbol{\alpha}}$ increases due to the rising lift forces toward the center of the channel, counteracting the initial decrease of the void fraction boundary layer.

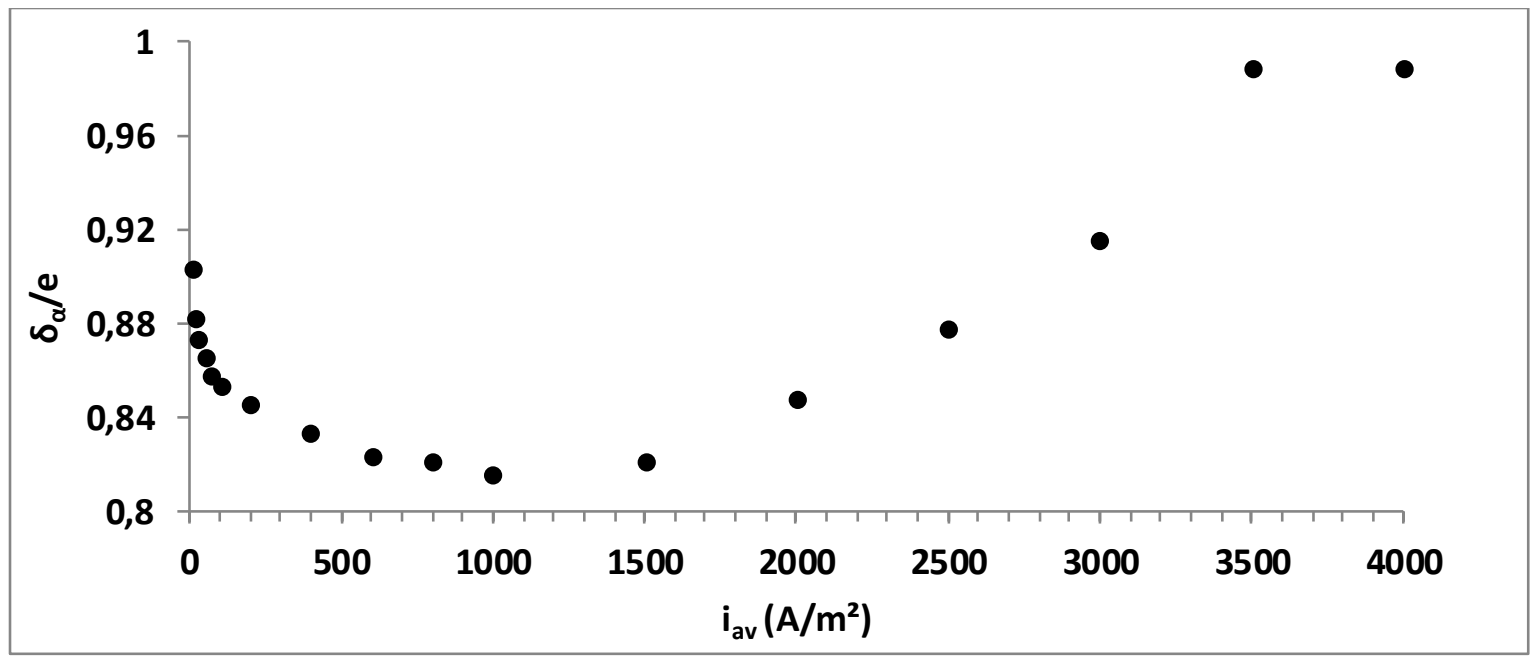

Fig. 6 : Relative plume thickness evolution, at low $\operatorname{Pr}_{\alpha}$, function of the mean current density.

\section{4 - Effect of forced convection}

Fig. 7 shows numerical results with several Poiseuille flow rates at the inlet of the channel, for various geometrical configurations and $\boldsymbol{P r}_{\boldsymbol{\alpha}}$ values. To highlight the effects of forced convection on bubble plume thickness, all the plotted results were obtained for an inlet velocity 10 times superior to the bubble- 
induced flow rate recorded with the same geometry, current density and $\boldsymbol{P r}_{\boldsymbol{\alpha}}$ value. Therefore, the buoyancy forces can be neglected. Fig. 7 exhibits $\frac{\boldsymbol{\delta}_{\alpha}}{\boldsymbol{e}}$ versus $\boldsymbol{P r}_{\boldsymbol{\alpha}} \boldsymbol{R}_{\boldsymbol{D}_{\boldsymbol{H}}}$ in log-scale. The dark circles and triangles were obtained respectively for $\boldsymbol{P r}_{\alpha}=\mathbf{0 . 1}$ and $\boldsymbol{P} \boldsymbol{r}_{\alpha}=\mathbf{1}$, with geometrical aspect $\frac{\boldsymbol{L}}{e}=\mathbf{7}$. For the same ratio, light circles and triangles represent respectively cases $\boldsymbol{P r}_{\boldsymbol{\alpha}}=\mathbf{1 0}$ and $\boldsymbol{P r}_{\boldsymbol{\alpha}}=\mathbf{8 0}$.

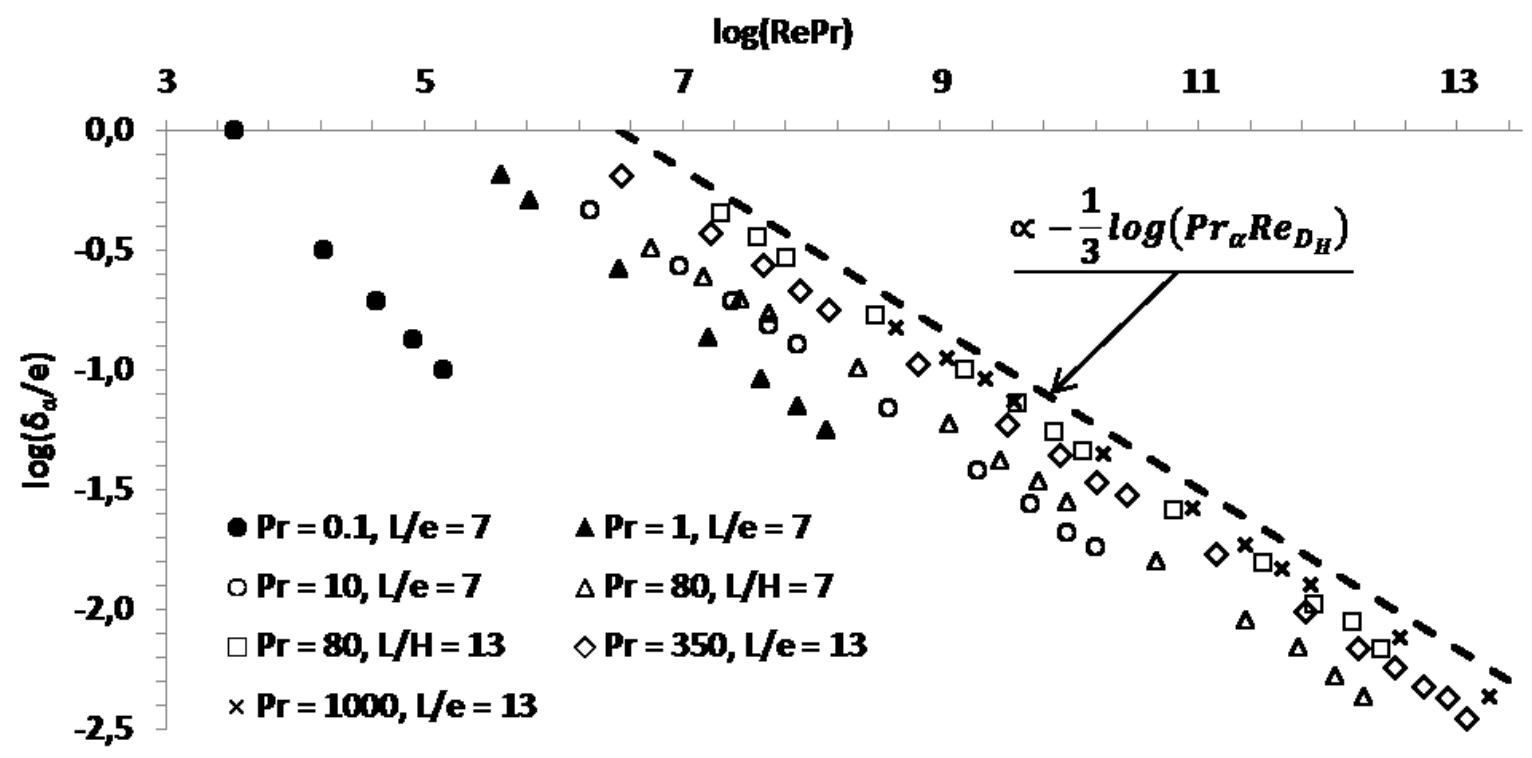

Fig. 7 : Effect of convection on plume thickness. For $10<\operatorname{Pr}_{\alpha}<1000$ (light marks) the slopes of linear regressions are -0.35 . For $\operatorname{Pr}_{\alpha}=1$ (dark triangles) the slope is -0.4 and for $\operatorname{Pr}_{\alpha}=0.1$ (dark circles), the slope is -0.6 .

Finally, light squares, diamonds and crosses result from simulations at $\frac{L}{e}=13$, with following Prandtl-analogous numbers: 80, 350 and 1000. The non-dimensional ratio $\frac{\delta_{\alpha}}{e}$ at high $\boldsymbol{P r _ { \alpha }}$ seems to be function of $\left(\operatorname{Pr}_{\alpha} \operatorname{Re}_{D_{H}}\right)^{-1 / 3}$, while for $\operatorname{Pr}_{\alpha}=1$ and $\operatorname{Pr}_{\alpha}=0.1$, the logarithmic-slopes are respectively -0.4 and -0.6 .

A scale analysis was achieved to clarify these relationships. Under the forced convection conditions, in the convection-dispersion balance (3.3), the velocity scale in the inner region of the boundary layer can be simply expressed by: $\boldsymbol{V} \sim \boldsymbol{v}_{\text {mean }} \frac{\boldsymbol{\delta}_{\alpha}}{\boldsymbol{e}}$. Therefore, (3.3) becomes:

$$
\frac{\delta_{\alpha}}{e} \sim\left(\frac{L}{e}\right)^{1 / 3}\left(\operatorname{Pr}_{\alpha} \operatorname{Re}_{D_{H}}\right)^{-1 / 3}
$$

As noticed previously, relation (3.9) agrees with the computed results at high Prandt/ values, assuming hydrodynamic self-diffusion control and the constant estimation of $\boldsymbol{P r}_{\boldsymbol{\alpha}}$ value. For lower Prandtl values, however, the competition of Saffman lift forces steered toward the wall explain the gradual decrease of slopes.

Standard alkaline water electrolysis operates typically at $\boldsymbol{P} \boldsymbol{r}_{\boldsymbol{\alpha}}>\mathbf{1}$, as in the experimental works of Boissonneau and Byrne $\left(\mathbf{8}<\boldsymbol{P r}_{\boldsymbol{\alpha}}<\mathbf{6 0}\right)$. The present model can predict reliably bubbles plume 
thickness evolution. However, a generic quantitative correlation cannot be defined because both the flow rate and the geometry impact the real Prandt/value, as emphasized in $\$ 3.2$.

These results demonstrate that an additional forced flow significantly reduces the bubble plume thickness. Indeed, when the natural flow rate is not sufficient to efficiently convey the bubbles out of the channel, forced flow can avoid mixing of the electrochemically generated gaseous species. In addition, an increasing laminar flow rate at the bottom of electrodes has several advantages. It decreases departing bubbles radius, thus reducing the dispersion. It lowers the resistivity of the mixture due to a lower averaged void fraction, higher electrode active surfaces and more uniform current density distributions. And it leads to a continuous reduction of void fraction boundary layer thickness, regardless to the prevailing dispersion mechanism (in opposition to the results obtained at high current density in bubbledriven convection).

\section{4- Conclusion}

A two-phase mixture model is used in this work to simulate the bubbly flow resulting from typical water electrolysis process. Various resolutions, using Comsol Multiphysics software, are performed under uniform electrical current density, in two dimensional laminar flow regimes between vertical electrodes. Comparisons with experimental results show the good applicability of the model for alkaline water electrolysis (small bubble diameter). By means of thermal analogy, two dimensionless numbers $\left(\boldsymbol{R} \boldsymbol{\alpha}_{\boldsymbol{f}, \boldsymbol{e}}=\right.$

$\frac{\boldsymbol{v}_{\boldsymbol{c}} \boldsymbol{U}_{\boldsymbol{g}} \boldsymbol{e}^{\mathbf{5}}}{\boldsymbol{r}^{\mathbf{6}} \mathbf{L g}}$ and $\left.\boldsymbol{P r}_{\boldsymbol{\alpha}}=\frac{\boldsymbol{v}_{C}}{\boldsymbol{K}_{\boldsymbol{\alpha}}}\right)$ are defined in order to characterize natural bubble-driven convection. For $\boldsymbol{P r}_{\boldsymbol{\alpha}} \geq$ $\mathbf{1}$, a scale analysis is performed to predict the evolution of the bubble plumes along the electrodes. It is observed that the most influent parameter is the bubble diameter, which depends on various parameters (e.g. electrolyte viscosity, pressure, current density...). Results also show that bubble dispersion mechanism depends strongly on flow conditions, imposed current density and physical parameters. This explains why further studies should be considered in order to define specific correlations for practical cases. A scale analysis is also performed in forced convection configuration and a scaling law for predicting the thickness gas thickness layer is proposed for $\boldsymbol{P} \boldsymbol{r}_{\boldsymbol{\alpha}} \geq \mathbf{1}$. The forced inlet velocity enhances the process performance in many aspects, mostly by reducing the bubbles plume thickness and avoiding the recombination of the electrochemically generated gaseous species.

\section{Acknowledgments}

This research was supported by AGIR (UJF/G-INP) $2013 S$ MI07. 


\section{Nomenclature}

\begin{tabular}{|c|c|c|c|}
\hline$A$ & void fraction scale & \multicolumn{2}{|c|}{$U_{\text {Stokes }}$ Stokes flux $\left(m \cdot s^{-1}\right)$} \\
\hline$D$ & non-dimensional hydrodynamic & $U_{r}$ & void fraction relative flux $\left(m . s^{-1}\right)$ \\
\hline & diffusion coefficient & $v$ & phase velocity $\left(m \cdot s^{-1}\right)$ \\
\hline$D_{H}$ & hydraulic diameter $(m)$ & \multicolumn{2}{|c|}{$v_{\text {Stokes }}$ Stokes velocity $\left(m \cdot s^{-1}\right)$} \\
\hline$e$ & half inter-electrode gap $(m)$ & $V$ & vertical velocity scale $\left(\mathrm{m} \cdot \mathrm{s}^{-1}\right)$ \\
\hline $\overrightarrow{e_{x}}, \overrightarrow{e_{y}}$ & horizontal and vertical unity vectors & $W$ & horizontal velocity scale $\left(m \cdot s^{-1}\right)$ \\
\hline$f$ & hindering function & $x$ & horizontal position (m) \\
\hline$F$ & Faraday constant $\left(\right.$ C. $\left.\mathrm{mol}^{-1}\right)$ & $y$ & vertical position $(m)$ \\
\hline$g$ & gravitational constant $\left(m \cdot s^{-2}\right)$ & & \\
\hline$H$ & inter-electrode gap $(m)$ & \multicolumn{2}{|c|}{ Greek symbols } \\
\hline$i$ & current density $\left(A . m^{-2}\right)$ & $\alpha$ & void fraction \\
\hline$i_{0}$ & exchanged current density $\left(A \cdot m^{-2}\right)$ & $\beta$ & non-dimensional shear-induced \\
\hline$i_{a v}$ & average current density $\left(A \cdot m^{-2}\right)$ & & diffusion coefficient \\
\hline$I$ & identity matrix & $\dot{\gamma}$ & shear rate $\left(s^{-1}\right)$ \\
\hline$K_{\alpha}$ & $\begin{array}{l}\text { void fraction dispersion coefficient } \\
\left(m^{2} . s^{-1}\right)\end{array}$ & $\delta_{\alpha}$ & $\begin{array}{l}\text { void fraction boundary layer thickness } \\
\text { (m) }\end{array}$ \\
\hline$L$ & electrode length $(m)$ & $\delta_{H}$ & hydrodynamic boundary layer thickness \\
\hline$L_{\text {in }}$ & laminar entrance length $(\mathrm{m})$ & & $(m)$ \\
\hline$p$ & absolute pressure $(\mathrm{Pa})$ & $\delta_{T}$ & thermal boundary layer thickness ( $m$ ) \\
\hline$P$ & reduced pressure $(P a)$ & $\eta$ & overpotential (V) \\
\hline$P r_{\alpha}$ & Prandtl-equivalent number & $\kappa$ & non-dimensional shear-induced \\
\hline$q$ & mass-averaged velocity $\left(\mathrm{m} . \mathrm{s}^{-1}\right)$ & & migration coefficient \\
\hline$r_{b}$ & bubble radius $(m)$ & $\mu$ & dynamic viscosity (Pa.s) \\
\hline$R$ & universal gas constant $\left(J \cdot \mathrm{mol}^{-1} \cdot \mathrm{K}^{-1}\right)$ & $v$ & kinematic viscosity $\left(m \cdot s^{-2}\right)$ \\
\hline$R e_{D_{H}}$ & channel Reynolds number & $\rho$ & density $\left(k g \cdot m^{-3}\right)$ \\
\hline$R \alpha_{f, e}$ & Rayleigh-equivalent number & $\sigma$ & electrolyte conductivity $\left(S \cdot m^{-1}\right)$ \\
\hline$T$ & operating temperature $(K)$ & $\tau$ & shear stress $(\mathrm{Pa})$ \\
\hline$U$ & superficial velocity $\left(m \cdot s^{-1}\right)$ & $\phi$ & electrolyte potential ( $V$ ) \\
\hline \multicolumn{4}{|c|}{$U_{\text {Hdiff }}$ hydrodynamic self-diffusion $\left(m \cdot s^{-1}\right)$} \\
\hline$U_{H 2}$ & hydrogen flux generation $\left(m \cdot \mathrm{s}^{-1}\right)$ & \multicolumn{2}{|c|}{ Subscripts } \\
\hline$U_{g}$ & gas flux generation $\left(m \cdot s^{-1}\right)$ & $C$ & continuous phase \\
\hline$U_{m i g}$ & shear-induced migration $\left(\mathrm{m} . \mathrm{s}^{-1}\right)$ & $D$ & dispersed phase \\
\hline$U_{O 2}$ & oxygen flux generation $\left(m \cdot s^{-1}\right)$ & $x$ & horizontal component \\
\hline$U_{\text {Saff }}$ & Saffman lift $\left(m \cdot s^{-1}\right)$ & $y$ & vertical component \\
\hline$U_{\text {Sdiff }}$ & shear-induced diffusion $\left(m \cdot s^{-1}\right)$ & & \\
\hline
\end{tabular}




\section{References}

[1] P. Boissonneau, P. Byrne, An experimental investigation of bubble -induced free convection in a small electrochemical cell, J. Appl. Electrochem. 30 (2000) 767-775. doi:10.1023/A:1004034807331.

[2] Q. Wang, B. Li, Z. He, N. Feng, Simulation of Magnetohydrodynamic Multiphase Flow Phenomena and Interface Fluctuation in Aluminum Electrolytic Cell with Innovative Cathode, Metall. Mater. Trans. B. 45 (2014) 272-294. doi:10.1007/s11663-013-0001-z.

[3] K. Wang, P. Pei, Z. Ma, H. Xu, P. Li, X. Wang, Morphology control of zinc regeneration for zinc-air fuel cell and battery, J. Power Sources. 271 (2014) 65-75. doi:10.1016/j.jpowsour.2014.07.182.

[4] K. Zeng, D. Zhang, Recent progress in alkaline water electrolysis for hydrogen production and applications, Prog. Energy Combust. Sci. 36 (2010) 307-326. doi:10.1016/j.pecs.2009.11.002.

[5] H. Vogt, The actual current density of gas-evolving electrodes-Notes on the bubble coverage, Electrochimica Acta. 78 (2012) 183-187. doi:10.1016/j.electacta.2012.05.124.

[6] N. Nagai, M. Takeuchi, T. Kimura, T. Oka, Existence of optimum space between electrodes on hydrogen production by water electrolysis, Int. J. Hydrog. Energy. 28 (2003) 35-41. doi:10.1016/S0360-3199(02)00027-7.

[7] Á. Frías-Ferrer, J. González-García, V. Sáez, C.P. de León, F.C. Walsh, The effects of manifold flow on mass transport in electrochemical filter-press reactors, AIChE J. 54 (2008) 811-823. doi:10.1002/aic.11426.

[8] K. Aldas, N. Pehlivanoglu, M. Mat, Numerical and experimental investigation of two-phase flow in an electrochemical cell, Int. J. Hydrog. Energy. 33 (2008) 3668-3675. doi:10.1016/j.ijhydene.2008.04.047.

[9] P. Mandin, A.A. Aissa, H. Roustan, J. Hamburger, G. Picard, Two-phase electrolysis process: From the bubble to the electrochemical cell properties, Chem. Eng. Process. Process Intensif. 47 (2008) 19261932. doi:10.1016/j.cep.2007.10.018.

[10] S.M. Ghiaasiaan, Two-phase flow, boiling and condensation in conventional and miniature systems, Cambridge University Press, New York, 2008

[11] E. Climent, J. Magnaudet, Large-Scale Simulations of Bubble-Induced Convection in a Liquid Layer, Phys. Rev. Lett. 82 (1999) 4827-4830. doi:10.1103/PhysRevLett.82.4827.

[12] M.C. Ruzicka, N.H. Thomas, Buoyancy-driven instability of bubbly layers: analogy with thermal convection, Int. J. Multiph. Flow. 29 (2003) 249-270. doi:10.1016/S0301-9322(02)00150-7.

[13] K. Iga, R. Kimura, Convection driven by collective buoyancy of microbubbles, Fluid Dyn. Res. 39 (2007) 68-97. doi:10.1016/j.fluiddyn.2006.08.003.

[14] H. Vogt, Heat transfer in boiling and mass transfer in gas evolution at electrodes - The analogy and its limits, Int. J. Heat Mass Transf. 59 (2013) 191-197. doi:10.1016/j.ijheatmasstransfer.2012.12.018.

[15] M. Ishii, T. Hibiki, Thermo-Fluid Dynamics of Two-Phase Flow, Springer New York, New York, NY, 2011.

[16] M. Ishii, N. Zuber, Drag coefficient and relative velocity in bubbly, droplet or particulate flows, AIChE J. 25 (1979) 843-855. doi:10.1002/aic.690250513.

[17] L.J.J. Janssen, C.W.M.P. Sillen, E. Barendrecht, S.J.D. van Stralen, Bubble behaviour during oxygen and hydrogen evolution at transparent electrodes in KOH solution, Electrochimica Acta. 29 (1984) 633-642. doi:10.1016/0013-4686(84)87122-4.

[18] A.A. Dahlkild, Modelling the two-phase flow and current distribution along a vertical gas-evolving electrode, J. Fluid Mech. 428 (2001) 249-272. doi:10.1017/S0022112000002639.

[19] R. Wedin, A.A. Dahlkild, On the Transport of Small Bubbles under Developing Channel Flow in a Buoyant Gas-Evolving Electrochemical Cell, Ind. Eng. Chem. Res. 40 (2001) 5228-5233. doi:10.1021/ie001073u. 
[20] H. Nicolai, B. Herzhaft, E.J. Hinch, L. Oger, E. Guazzelli, Particle velocity fluctuations and hydrodynamic self-diffusion of sedimenting non-Brownian spheres, Phys. Fluids 1994-Present. 7 (1995) 12-23. doi:10.1063/1.868733.

[21] P.G. Saffman, The lift on a small sphere in a slow shear flow, J. Fluid Mech. 22 (1965) 385-400. doi:10.1017/S0022112065000824.

[22] D. Leighton, A. Acrivos, Measurement of shear-induced self-diffusion in concentrated suspensions of spheres, J. Fluid Mech. 177 (1987) 109-131. doi:10.1017/S0022112087000880.

[23] U. Schaflinger, Centrifugal separation of a mixture, Fluid Dyn. Res. 6 (1990) 213-249. doi:10.1016/0169-5983(90)90014-P.

[24] T. Isono, Density, Viscosity, and Electrolytic Conductivity of Concentrated Aqueous Electrolyte Solutions at Several Temperatures. Alkaline-Earth Chlorides, $\mathrm{LaCl} 3, \mathrm{Na} 2 \mathrm{SO} 4, \mathrm{NaNO} 3, \mathrm{NaBr}, \mathrm{KNO} 3$, $\mathrm{KBr}$, and $\mathrm{Cd}(\mathrm{NO} 3) 2,(1984)$.

[25] A. Bejan, CONVECTION HEATTRANSFER, 3RD ED, Wiley India Pvt. Limited, 2006.

[26] A.-M. Dalbert, F. Penot, J.-L. Peube, Convection naturelle laminaire dans un canal vertical chauffe a flux constant, Int. J. Heat Mass Transf. 24 (1981) 1463-1473. doi:10.1016/0017-9310(81)90214-3.

[27] Z.D. Wei, M.B. Ji, S.G. Chen, Y. Liu, C.X. Sun, G.Z. Yin, et al., Water electrolysis on carbon electrodes enhanced by surfactant, Electrochimica Acta. 52 (2007) 3323-3329. doi:10.1016/j.electacta.2006.10.011. 